\title{
Costo/efficacia della prevenzione di infezioni alle prime vie aeree mediante un estratto batterico immunostimolante aspecifico (OM-85)
}

Orietta Zaniolo*, Lorenzo Pradelli*, Mario Eandi $\S$

\begin{abstract}
OBJECTIVES: To estimate the pharmacoeconomical impact of preventing recurrent upper respiratory tract infections (URTIs) with OM-85, a non-specific immunostimulating agent, in at-risk children.

METHODS: Implementation of a decisional model. The evaluation of effectiveness (number of prevented URTIs/therapeutic cycle) was based on weighted average of the results of four randomized, double-blind, placebo-controlled trials identified by literature reviewing. The clinical events considered in the model were natural resolution of the infection, onset of complications (acute otitis media, sinusitis, others) and their evolution. Baseline event probabilities were derived by reviewing published data in the literature. URTI-related direct and indirect costs supported by patient, by Italian health system and by community were structured according with the principal guidelines and implemented with current Italian prices and tariffs. The cost/ effectiveness of OM- 85 was calculated for five scenarios, differing in the number of therapeutic cycles, grade of patient co-payment and other secondary assumptions. Sensibility analyses were performed to evaluate the model robustness.

RESULTS: Immunostimulation with one cycle of OM- 85 prevented on average 1,60 URTI/patient in six months $(\mathrm{RR}=0,515)$. In the basic scenario, this preventive action induced savings for $107,42 \mathrm{euro} /$ patient in the perspective of the patient's family, for 48,52 euro/patient in the perspective of Italian health system and for 231,26 in the community perspective. Sensibility analyses confirmed the robustness of basic scenario results. Threshold analyses showed that OM-85 prophylaxis is economically convenient as long as more than $7 \%$ of infections are prevented or global cost of one episode of URTI is greater than 10,00 euro. CONCLUSIONS: Non-specific immunotherapy with OM-85 induces a reduction in the incidence of URTIs in atrisk children with a concurrent saving for patient and health system.
\end{abstract}

Keywords: OM-85, cost/effectiveness, upper respiratory tract infections (URTIs) Farmeconomia e percorsi terapeutici 2005; 6 (3): 169-184

\section{INTRODUZIONE}

La rinofaringite acuta, o comune raffreddore, consiste in un'infiammazione delle vie aeree superiori che interessa, in vario grado, il naso, i seni paranasali, la gola, la laringe $e$, in qualche caso, la trachea e i bronchi.

In generale le infezioni delle vie aeree superiori (Upper Respiratory Tract Infection URTI) sono ad eziologia virale; la comparsa di complicanze è invece solitamente un problema batterico e riguarda principalmente lo sviluppo di otite media acuta e sinusite.

La comparsa in infezioni ricorrenti segna l'adattamento del bambino all'ambiente circostante e la conseguente acquisizione delle difese immunitarie. Alcuni bambini però, per motivi non ancora chiariti, acquisiscono tali difese meno rapidamente di altri e presentano una forma ricorrente di URTI; un bambino è ritenuto affetto da URTI ricorrente se presenta almeno tre nuovi episodi in un periodo di 6 mesi.

Questa condizione rappresenta un problema rilevante sia da un punto di vista clinico che socio-economico e quindi richiede, nella gran parte di casi, la messa a punto da parte del medico di una strategia preventiva.

Le prime azioni da intraprendere riguardano le condizioni di vita del bambino. Favorire l'allattamento al seno, accudire il bambino a casa, ridurre il fumo passivo e, dove possibile, l'inquinamento in generale, rappresentano provvedimenti utili per diminuire il rischio di contrarre malattie infettive e, in particolare, di tipo respiratorio.
*Advanced Research Srl

$\S$ Dipartimento di Farmacologia clinica, Università di Torino 
Inoltre vi sono alcuni disturbi, come ad esempio le adenoidi ingrossate, la deficienza di ferro, la rinite allergica, il reflusso gastroesofageo, che costituiscono fattori di rischio per la comparsa di nuovi episodi di URTI e che, quindi, occorre modificare.

Da un punto di vista epidemiologico, però, è stato dimostrato che la comparsa di infezioni respiratorie recidivanti è spesso associata ad un deficit immunitario che interessa le $\mathrm{IgG}$ e le IgA [1]; ne consegue che la somministrazione di un immunostimolante, in concomitanza con il miglioramento dello stile di vita e la correzione dei fattori di rischio, rappresenta il trattamento preventivo più indicato.

Fra i composti in grado di stimolare le difese immunitarie, OM-85 (Broncho Munal ${ }^{\circledR}$ ) è un lisato batterico liofilizzato ottenuto da otto batteri: Haemophilus influenzae, Diplococcus pneumoniae, Klebsiella pneumoniae e ozaenae, Staphylococcus aureus, Streptococcus pyogenes e viridans, Neisseria catarrhalis. Esso stimola la risposta immunitaria locale mediante l'attivazione del tessuto linfoide della mucosa, dei macrofagi e dei fagociti; inoltre stimola l'attività citotossica e la produzione di IgA salivare e broncoalveolare, così come di IgA e IgG sieriche [2, 3].

Numerosi studi in doppio cieco hanno dimostrato la sua efficacia nel ridurre la frequenza delle infezioni a carico di naso, gola e orecchio sia ad eziologia batterica che virale, con conseguente diminuzione del consumo di antibiotici e di altre terapie associate.

Scopo di questo lavoro è l'implementazione di un modello farmacoeconomico che permetta di calcolare il risparmio indotto dall'utilizzo di OM-85 nella prevenzione di recidive di URTI nei bambini a rischio.

L'analisi dei costi è stata svolta utilizzando la prospettiva del paziente, del Sistema Sanitario Nazionale e della società.

\section{METODI}

\section{Revisione della letteratura}

Per rintracciare i principali articoli riguardanti le infezioni respiratorie ricorrenti, le relative complicazioni e l'efficacia di OM-85 nella prevenzione delle recidive è stata condotta una ricerca sulla banca dati Medline e del sito del Ministero della Salute Italiano; inoltre sono state analizzate le voci bibliografiche degli articoli trovati e gli abstract di congressi e conferenze svolti sull'argomento.

Le parole chiave medianti le quali è stata svolta la ricerca sono state: "Upper respiratory infections", "bacterial lysate", "ear, nose, throat diseases". In tutto sono state analizzate circa 150 voci bibliografiche.

Al fine di valutare l'efficacia del lisato batterico nella prevenzione di URTI in età pediatrica, fra gli studi analizzati sono stati presi in considerazione solo i trial randomizzati in doppio cieco e controllati, svolti su pazienti di età compresa fra 0 e 19 anni a cui era stato somministrato OM-85 secondo un determinato schema terapeutico.

Considerando che il farmaco è indicato nel trattamento preventivo di pazienti con infezioni ricorrenti delle vie aeree superiori, gli studi selezionati per la valutazione dell'efficacia dovevano avere i seguenti criteri di inclusione:

- $>$ o $=$ tre URTI nel periodo autunno-invernale o nei sei mesi precedenti al trial (in accordo con la definizione di paziente a rischio che giustifica l'uso di una terapia preventiva [4]);

- no immunodeficienza;

- no allergia ai prodotti di origine batterica;

- no gravi comorbilità, es. disturbi respiratori cronici, insufficienza cardiaca, diabete mellito;

- no trattamento con corticosteroidi, immunostimolanti, immunosoppressori nei precedenti sei mesi.

Sono stati inclusi nell'analisi soltanto gli studi che avevano come outcome clinico il numero medio di infezioni per paziente durante il periodo considerato.

In base a questi criteri sono stati selezionati tre studi della durata di sei mesi in cui è stato somministrato un solo ciclo di OM-85 e uno studio della durata di 12 mesi con due cicli di somministrazione.

Ogni ciclo di terapia comprende la somministrazione di una capsula da $3,5 \mathrm{mg} /$ die di OM85 per i primi 10 giorni di ogni mese per tre mesi consecutivi, seguiti da tre mesi di follow up.

Le principali caratteristiche di questi studi sono riassunti in Tabella I.

Per ottenere 1'efficacia media della prevenzione con OM-85 il risultato di ciascun trial è stato pesato per la dimensione totale della rispettiva coorte.

Alcuni studi hanno valutato anche la differenza tra gruppo trattato e gruppo placebo in termini di gravità di sintomi e di complicanze, di durata della terapia e di giorni persi di scuola; considerata però la disomogeneità dei risultati ottenuti, abbiamo conservativamente assunto che la sola differenza tra le due strategie (vaccino vs. placebo) consistesse nel numero di URTI riportate dal paziente in un determinato periodo di tempo (6 o 12 mesi).

Oltre a questo scenario base, si è poi ipotizzato uno scenario alternativo (scenario 2, Tabella IV, V e VI) in cui la percentuale di episodi complicati cambia a seconda dell'utilizzo o meno della terapia preventiva; infatti secondo i dati risultanti dallo studio svolto da Jara-Pérez e colleghi, in seguito a somministrazione del vaccino il numero di URTI complicati 
è sceso del 41,1\%. Applicando questa variazione al modello economico si può avere un'idea del risparmio ottenibile qualora la terapia preventiva agisca anche sul decorso dell'episodio e non soltanto sul numero di episodi per periodo.

\section{Descrizione del modello farmacoeconomico}

Il modello farmacoeconomico calcola il costo per paziente nel caso in cui egli venga trattato preventivamente con $\mathrm{OM}-85$, confrontandolo con il costo associato a nessuna terapia preventiva, durante un periodo di sei mesi o di un anno.

Il modello è stato realizzato mediante l'utilizzo del software TreeAge - DATA Pro / 4.0.

Gli eventi clinici da considerare durante un'infezione alle vie aeree superiori sono sostanzialmente: il decorso naturale della condizione infettiva iniziale, le complicanze acute associate all'infezione virale e il loro decorso.

Le complicanze batteriche locali associate al comune raffreddore sono rappresentate soprattutto dall'otite media acuta (OMA) e dalla sinusite acuta; laringite, tonsillite, bronchite ecc costituiscono le altre complicanze infettive abituali $[4,9,10]$.

Come si può osservare in Figura 1, il nodo decisionale (quadrato) rappresenta l'alternativa trattamento preventivo/nessun trattamento preventivo, dopodiché la progressione clinica è uguale nei due rami (pallino $=$ chance node). L'episodio può avere un decorso naturale (URTI NC) oppure può complicarsi (URTI $\mathrm{CC})$. A loro volta le due complicanze più frequenti, sinusite e otite media, possono risolversi mediante la somministrazione di antibio-

\begin{tabular}{|c|c|c|c|c|}
\hline Autore & $\begin{array}{l}\text { Durata } \\
\text { studio }\end{array}$ & $\begin{array}{l}\text { Età dei } \\
\text { pazienti }\end{array}$ & $\begin{array}{l}\text { Dimensione } \\
\text { della coorte }\end{array}$ & $\begin{array}{l}\text { N. medio di episodi } \\
\text { acuti per paziente * }\end{array}$ \\
\hline \multicolumn{5}{|c|}{1 ciclo di somministrazione } \\
\hline $\begin{array}{l}\text { Maestroni et al. } \\
\quad(1984)[5]\end{array}$ & $\begin{array}{l}6 \text { mesi } \\
3+3\end{array}$ & 1-16 anni & $\begin{array}{l}\text { PZ: } 20 \\
\text { O: } 11 \\
\text { P: } 9\end{array}$ & $\begin{array}{l}\text { O: } 1,9 \\
P: 5,4\end{array}$ \\
\hline $\begin{array}{l}\text { Paupe } \\
(1991)[6]\end{array}$ & $\begin{array}{l}6 \text { mesi } \\
3+3\end{array}$ & 0,5-19 anni & $\begin{array}{c}\text { PZ: } 116 \\
\text { O: } 61 \\
\text { P: } 55\end{array}$ & $\begin{array}{l}\text { O: } 2,066 \\
P: 3,509\end{array}$ \\
\hline $\begin{array}{l}\text { Jara-Pérez et al. } \\
\text { (2000) [7] }\end{array}$ & $\begin{array}{l}6 \text { mesi } \\
3+3\end{array}$ & 6-13 anni & $\begin{array}{c}\text { PZ: } 199 \\
\text { O: } 99 \\
\text { P: } 100\end{array}$ & $\begin{array}{l}\text { O: } 1,44 \\
\text { P: } 2,99\end{array}$ \\
\hline \multicolumn{5}{|c|}{2 cicli di somministrazione } \\
\hline $\begin{array}{c}\text { Gutiérrez- } \\
\text { Tarango et al. } \\
\text { (2001) [8] }\end{array}$ & $\begin{array}{l}12 \text { mesi } \\
(3+3)\end{array}$ & 1-12 anni & $\begin{array}{l}\text { PZ: } 54 \\
\text { O: } 26 \\
\text { P: } 28\end{array}$ & $\begin{array}{l}\text { O: } 5,04 \\
P: 8\end{array}$ \\
\hline
\end{tabular}

$\mathrm{PZ}=$ pazienti, $\mathrm{O}=\mathrm{OM}-85, \mathrm{P}=$ placebo, ${ }^{*}$ Nel periodo di durata del trial

\section{Tabella I}

Caratteristiche dei trial selezionati per la valutazione dell'efficacia di OM-85 e relativi schemi terapeutici

tici di prima linea (Risp ab 1' linea) oppure richiedere l'utilizzo di una terapia di seconda scelta; anche in questo caso la complicanza può risolversi (Risp ab 2' linea) o richiedere ulteriori interventi (No risp).

Il payoff (termine usato nel programma per indicare il valore netto relativo ad uno specifico scenario o outcome) corrisponde al costo associato ad un determinato nodo terminale (triangolo) che, a seconda della prospettiva assunta, corrisponderà al costo a carico del SSN, del paziente o della società.

\section{Probabilità degli eventi nel modello}

La probabilità associata al verificarsi di un evento è stata stimata in base a dati pubblicati

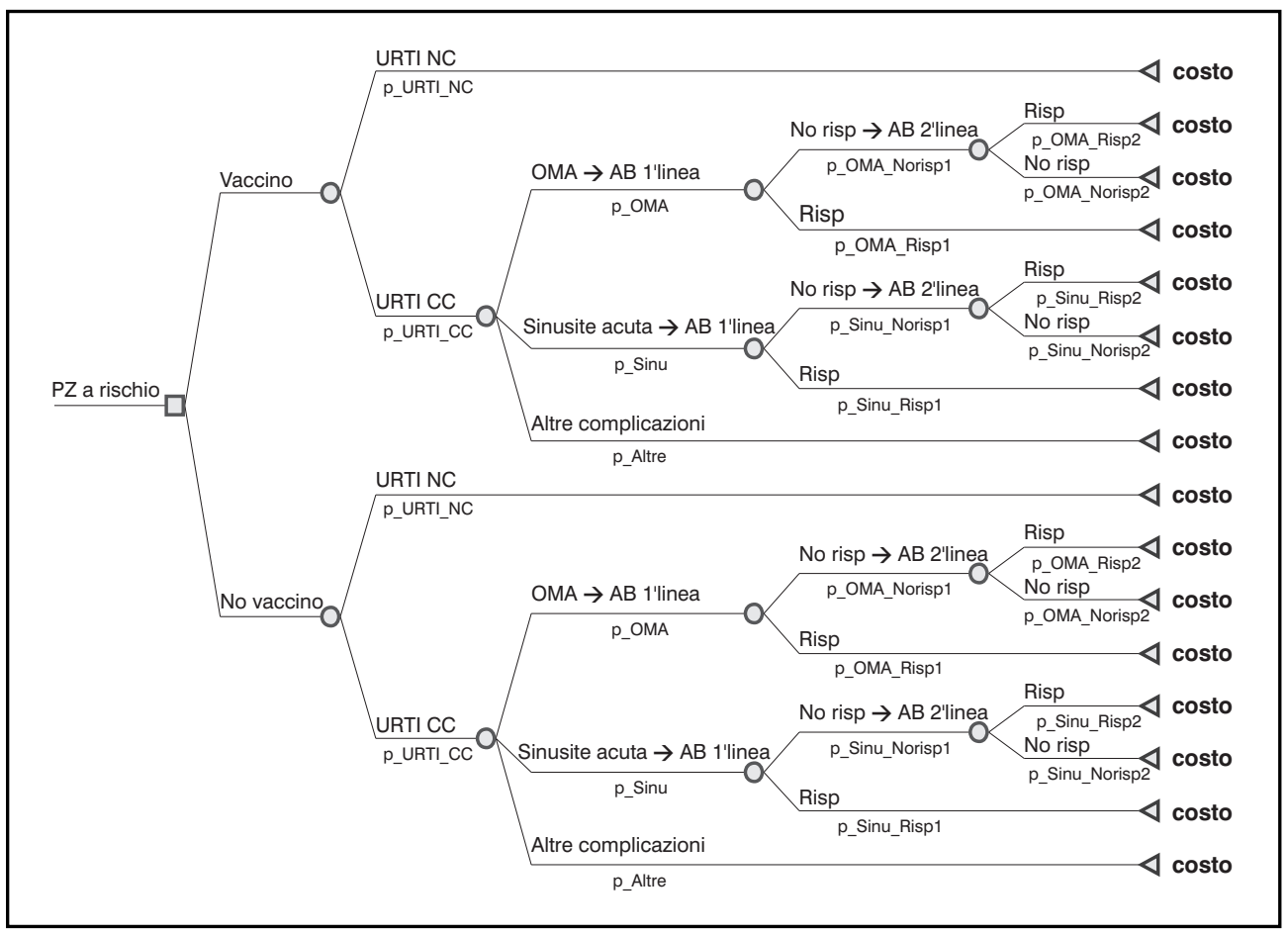

Figura 1

Modello

farmacoeconomico

con progressione clinica di un URTI

$\mathrm{PZ}=$ paziente

$\mathrm{NC}=$ non complicata

$\mathrm{CC}=$ con complicanze

$\mathrm{ab}=$ antibiotico

$\mathrm{p}=$ probabilità 


Costo totale $=$ costo vaccino + costo periodo considerato
Costo periodo $=$ costo episodio $\times$ numero episodi nel periodo considerato
Costo episodio $=$ costi diretti + costi indiretti
Costi diretti $=$ costi farmaceutici + costi visite + costi ospedalizzazione
Costi farmaceutici $=$ costi antibiotici + costi farmaci sintomatici

Tabella II

Struttura dei costi

* Broncho Munal ${ }^{\circledR}$ Abiogen Pharma in letteratura, riportati nelle linee guida o nelle banche dati di associazioni pediatriche.

Il $69 \%$ dei casi di infezione alle vie aeree superiori ha un decorso favorevole non complicato senza trattamento antibatterico [11-13].

Secondo lo studio di van Cauwenberge circa il $75,3 \%$ dei casi di URTI non complicata richiede una visita pediatrica [9].

Nello scenario 2 è stato assunto che la percentuale di URTI complicati nel gruppo trattato diminuisca del $41,1 \%$ rispetto al gruppo controllo, come riportato nel trial svolto da JaraPerez e colleghi (18,3\% rispetto a $31 \%$ dello scenario base).

Secondo i dati riportati da van Cauwenberge, un episodio di URTI è complicato da otite media con una frequenza che va dal $7 \%$ al $22 \%$ dei casi; un altro studio rileva invece una frequenza di comparsa di OMA pari al 29,2\% dei casi di URTI [11]. In base a questi dati, per tale complicanza è stata assunta una frequenza media del $21,5 \%$.

La frequenza della sinusite acuta come complicanza di URTI varia da $0,5 \%$ a $10 \%$ secondo alcuni dati reperiti in letteratura $[10,14]$ e da $4 \%$ a $7,3 \%$ secondo dati provenienti da altri studi [11]; mediante il calcolo della media ponderata di tali valori è stata assunta una frequenza di comparsa di sinusite acuta del 5,5\%.

Complessivamente le altre complicanze compaiono nel 4\% circa dei casi di URTI $[9,11,12]$. Singolarmente, ognuna di queste complicanze (tonsillite, laringite, bronchite, ecc), ha un'incidenza significativamente minore rispetto ai casi di OMA o di sinusite; per tale ragione è stato assunto che si risolvano con la terapia indicata come prima scelta dalle principali linee guida, in quanto la probabilità di ulteriore aggravamento o di ospedalizzazione risulterebbe trascurabile rispetto agli altri eventi modellizzati.

Circa l' $80 \%$ dei casi di sinusite acuta risponde al trattamento antibiotico di prima linea $[15,16]$, nei restanti casi, è stato assunto in base ai dati pubblicati in letteratura che il trattamento di seconda linea abbia una percentuale di successo del 70\% [10,17]

L' $85 \%$ dei casi di otite media acuta risponde al trattamento antibiotico di prima linea [1821]; nel restante $15 \%$ dei casi, si è assunto che la terapia di seconda linea abbia una percentuale di successo del 70\% [18].
Secondo alcuni studi riportati in letteratura, circa il 3\% dei casi di OMA e di sinusite acuta in età pediatrica richiede il ricovero in ospedale [22-31]; in base a questo dato è stato assunto che circa il $65 \%$ delle OMA non rispondenti al trattamento di seconda linea e il $50 \%$ delle sinusiti con uguale decorso si risolvesse con l'ospedalizzazione.

In caso di URTI complicata le linee guida raccomandano una visita pediatrica; la visita viene solitamente ripetuta nel caso in cui il quadro clinico non migliori in seguito a somministrazione del trattamento antibiotico di prima linea. Qualora non si sia riscontrato un miglioramento neanche dopo la somministrazione di una seconda terapia antibiotica, le linee guida consigliano la consultazione di uno specialista $[15,16,32,33]$.

\section{Parametri farmacoeconomici}

Sono stati considerati i costi diretti e indiretti che il nucleo familiare del paziente, il Sistema Sanitario Nazionale e la collettività devono sostenere in media per ogni paziente con URTI ricorrente (Tabella II).

Il numero medio di episodi per paziente sarà diverso a seconda che si consideri il gruppo trattato o il gruppo placebo e a seconda che si stia considerando uno scenario ( 1 ciclo di trattamento) o l'altro (2 cicli di trattamento).

Il costo del vaccino nel ramo placebo sarà ovviamente uguale a zero.

A seconda della prospettiva assunta alcune di queste voci verranno o meno considerate o assumeranno valori diversi.

\section{Costo vaccino}

Un ciclo di trattamento preventivo con lisato batterico polivalente OM-85 comprende la somministrazione di una capsula da $3,5 \mathrm{mg} /$ die di OM-85 per i primi 10 giorni di ogni mese per tre mesi consecutivi, seguiti da tre mesi di follow up.

Una confezione di OM-85* da $30 \mathrm{cps}$ ad uso pediatrico $(3,5 \mathrm{~g}$ ognuna) soddisfa la richiesta posologica di un intero ciclo di vaccino e il suo prezzo al pubblico è pari a 17,80 euro [34]. Nello scenario in cui è stato assunto che il paziente ricevesse due cicli di vaccino, tale costo è risultato essere di 35,60 euro.

Il lisato batterico è totalmente a carico del paziente; esso rappresenta quindi un costo conteggiato nella prospettiva del nucleo familiare e della società e, chiaramente, solo per la coorte di pazienti vaccinati.

\section{Costo episodio: costi indiretti}

Raffreddore e infezioni respiratorie nei pazienti pediatrici rendono spesso indispensabile l'astensione dal lavoro di uno dei due genitori, tradizionalmente la madre. La perdita di giornate produttive e l'utilizzo di permessi non retribuiti rappresentano la voce più sostanzio- 
sa dei costi complessivi con serie ripercussioni sociali, in ogni Paese.

Secondo uno studio svolto da Cauwenberge e colleghi [9] al fine di determinare l'impatto economico delle rinofaringiti acute in bambini italiani e francesi, in media circa il 34,2\% dei casi di rinofaringite (complicata o meno) richiede la perdita di giorni lavorativi da parte di uno dei due genitori. Altri studi hanno confermato questo dato e hanno stimato il numero medio di giorni persi in 3,5 $\pm 1,5$ giorni [35-38].

Nonostante negli ultimi anni la trasformazione dei ruoli sociali abbia indotto, anche dal punto di vista legale e sociale, una maggiore partecipazione del padre alla vita del bambino (usufruendo per esempio di permessi di paternità), nella maggior parte dei casi è ancora la madre ad astenersi dal lavoro per prestare le cure e l'assistenza necessarie al proprio bambino. Per tale ragione i costi indiretti che gravano sulla collettività sono stati calcolati moltiplicando la retribuzione lorda giornaliera percepita in media da una lavoratrice di sesso femminile [39] per i giorni persi in media (3,5 gg di assenza) per la probabilità che l'evento accada $(0,342)$. Nella prospettiva del nucleo familiare, il costo indiretto medio per ogni episodio di infezione respiratoria è stato valutato in base alla retribuzione netta, stimata considerando una pressione fiscale media complessiva del 40\% (Vedere appedice - Calcolo dei costi indiretti medi per episodio).

E stato ipotizzato inoltre uno scenario in cui i costi da mancato guadagno non sono stati considerati, in modo da valutare la costo/ efficacia in funzione dei soli costi diretti (scenario 4, Tabella IV).

\section{Costo episodio: costi diretti}

Sono stati analizzati i costi farmaceutici per la cura di ogni episodio e relative complicanze, la spesa relativa a visite mediche e specialistiche e i costi di ospedalizzazione in caso di ulteriore aggravamento della complicanza.

Considerato che la coorte di pazienti analizzata ha un'età variabile fra i 6 mesi e i 19 anni, la quantità media di farmaco assunta giornalmente è stata ottenuta moltiplicando la dose $/ \mathrm{kg}$ suggerita nelle linee guida per il peso medio di un bambino di 9,5 anni. Il valore di riferimento è stato stimato dalla SINU (Società Italiana di Nutrizione Umana) e corrisponde a circa $30 \mathrm{~kg}$ [40].

Il costo dei farmaci è stato stimato in base ai prezzi d'acquisto delle forme farmaceutiche disponibili, dando maggior rilevanza all'alternativa più economica e con un confezionamento coerente con l'esigenza posologica.

\section{Costi diretti associati a URTI non}

\section{complicata}

Secondo le principali linee guida di pratica clinica, il comune raffreddore, essendo solitamente un'infezione di origine virale, non richie- de la somministrazione di una terapia antibiotica [41].

I sintomi solitamente includono rinorrea, tosse moderata, bruciore di gola, diminuzione dell'appetito, mal di testa e malessere generale. La febbre è presente di norma per un periodo di circa tre giorni e generalmente non eccede i $38,7^{\circ} \mathrm{C}$.

In caso di febbre o di dolore persistente, le linee guida consigliano l'assunzione di paracetamolo nella dose di $10-15 \mathrm{mg} / \mathrm{kg} / \mathrm{dose}$ ogni $4 / 6$ ore [42].

Per quanto concerne l'utilizzo di farmaci OTC (Over-the-counter) nel trattamento di sintomi quali rinorrea, tosse e congestione delle mucose nasali, le linee guida raccomandano di non eccedere con la somministrazione di tali medicinali ai bambini.

Secondo alcuni studi però, circa il $65 \%$ dei bambini con raffreddore fa uso di decongestionanti nasali e di soluzioni saline per irrigazioni nasali $[7,9,35,43]$.

\section{- Prospettiva del paziente}

Il paracetamolo, non essendo prescrivibile in regime di rimborsabilità, è a carico del paziente e il suo costo è stato stimato essere pari a 2,50 euro per episodio.

La spesa per decongestionanti nasali e soluzioni saline, calcolata in base alla media dei prezzi d'acquisto dei farmaci maggiormente richiesti in farmacia (clonazolina+fluocinolone, xylometazolina, vari tipi di acqua marina sterile e isotonica, acqua sulfurea), è di circa 7,00 euro per episodio. Ad ogni episodio di URTI non complicata corrisponderà quindi una spesa per farmaci OTC pari a questo valore moltiplicato per la probabilità che essi vengano utilizzati (vedere appendice - Calcolo del costo farmaci sintomatici).

\section{- Prospettiva del SSN}

Il Sistema Sanitario Nazionale remunera i medici e i pediatri di famiglia sostanzialmente su base capitaria con particolare riferimento al numero di assistiti; di conseguenza, il numero totale delle visite svolte non influisce sulla sua remunerazione [44]. In pratica, almeno nel breve termine, una visita addizionale erogata ai pazienti ha per il SSN un costo nullo. Secondo la logica del costo-opportunità, sulla quale si dovrebbe basare in ambito sanitario la scelta tra differenti alternative, le risorse allocate in una data attività hanno, invece, un valore pari a tutto ciò che si sarebbe potuto conseguire utilizzando tali risorse nel loro migliore impiego alternativo; in altre parole il tempo e le energie impiegate dal pediatra per visitare un paziente rappresentano fattori sottratti ad altre attività $\mathrm{o}$ ad altri pazienti. Nel lungo periodo questo può rappresentare un costo o un risparmio per il SSN.

Tale costo teorico è stato stimato sulla base di dati derivanti da un precedente studio svol- 
to al fine di quantificare il costo di tali visite nell'ambito del Sistema Sanitario Italiano; secondo questo studio una visita ambulatoriale del medico di medicina generale ha un costo medio pari a 12,32 euro [44]; i costi di farmacia a carico del SSN risultano pari a 0 in quanto $\mathrm{i}$ medicinali utilizzati nel trattamento di URTI non complicata non sono rimborsati dal Sistema Sanitario.

Parallelamente è stato ipotizzato uno scenario in cui il costo per visita pediatrica è considerato pari a 0 (scenario 4, Tabella V; scenario 3, Tabella VI).

\section{- Prospettiva della società}

Il costo diretto che grava sulla collettività in caso di URTI non complicata comprende sia i costi farmaceutici sostenuti dal nucleo familiare e sia il costo della visita pediatrica.

Costi diretti associati ai casi di complicanza rispondente a terapia di prima linea

Secondo le principali linee guida, l'otite media acuta e la sinusite sono due complicanze di origine batterica e richiedono un trattamento antibatterico adeguato; in base a tali raccomandazioni, l'amoxicillina rappresenta il trattamento di prima scelta, sostituito da trimetoprim/ sulfametossazolo in caso di accertata allergia alle penicilline.

Secondo alcune stime riportate in letteratura, circa il 3\% dei pazienti risulta allergico alle penicilline [45]; questo dato, che in un primo tempo è stato considerato nel calcolo, comporta una differenza in termini di costo irrilevante; per tale ragione è stato assunto che nessuno dei pazienti fosse allergico alle penicilline.

Dose e tempi di somministrazione sono stati assunti in base a quanto riportato nelle principali linee guida $[15,16,32,33,41,46-49]$.

Per quanto concerne le altre complicanze (laringite, tonsillite, bronchite, ecc.), nel tentativo di ottenere un'unica voce di costo che racchiudesse le spese relative alle diverse patologie, sono state adottate come riferimento le linee guida relative al trattamento di bronchite e di tonsillite [41,50,51]. Queste raccomandano l'uso di amoxicillina nel trattamento della tonsillite, mentre ne sconsigliano la prescrizione in caso di bronchite, dal momento che nel $90 \%$ dei casi la sua origine non è batterica. Per tale complicanza sono frequentemente prescritti farmaci mucolitici ed espettoranti (ambroxolo, acetilcisteina, carbocisteina, neltenexina) [50].

\section{- Prospettiva del paziente}

L'amoxicillina è un farmaco prescritto in regime di rimborsabilità da parte del $\mathrm{SSN}$, di conseguenza il suo costo d'acquisto non è a carico del paziente; tuttavia in circa 10 regioni italiane tutti i cittadini, con esclusione di invalidi di guerra, invalidi per servizio e pensionati esentati per limiti di reddito, devono partecipare alla spesa farmaceutica con il pagamento di un ticket che nella maggior parte di queste regioni è pari a 2,00 euro a confezione.

Il costo sostenuto dal nucleo familiare per 1'acquisto di amoxicillina per il trattamento di OMA e sinusite acuta è risultato rispettivamente di 4,00 euro (due confezioni) e 2,00 euro (una confezione) (vedere appendice - Calcolo del costo di amoxicillina).

Il costo per episodio è stato calcolato anche ipotizzando che il paziente viva in una regione che non applica forme di ticket (scenario 3, Tabella IV e V).

In caso di sinusite, è stato inoltre quantificato l'utilizzo di farmaci sintomatici come decongestionanti nasali e soluzioni saline per irrigazioni nasali $(7,00$ euro; $p=1)[7,9,35,43]$.

I farmaci mucolitici ed espettoranti utilizzati in caso di bronchite non sono rimborsati dal SSN (classe "C") e pertanto il loro costo medio, pari a circa 6,50 euro per episodio, è a carico del paziente.

Il costo sostenuto dal paziente per l'acquisto di amoxicillina in caso di tonsillite è invece pari a 2,00 euro, equivalenti al ticket relativo all'acquisto di una confezione (vedere appendice - Calcolo del costo di amoxicillina).

Il costo farmaceutico di un episodio di "altra complicanza" dipende quindi da questi due costi e dalle probabilità a loro associate $(\mathrm{p}=$ 0,5 , assumendo che tonsillite e bronchite si verifichino con uguale probabilità) (vedere appendice - Calcolo del costo farmaci sintomatici e Calcolo del costo di amoxicillina).

Nel computo dei costi farmaceutici inerenti a tutte le complicanze risolte con terapia di prima scelta è stato inserito anche il costo di 2,50 euro relativo all'utilizzo di paracetamolo, raccomandato come antipiretico e antidolorifico dalle principali linee guida.

\section{- Prospettiva del SSN}

Il costo sostenuto dal Sistema Sanitario per ogni episodio di complicanza risolta con il trattamento antibiotico di prima scelta è costituito, oltre che dal costo di una visita pediatrica, dal costo di amoxicillina scorporato della quota di ticket con la quale il paziente partecipa alla spesa farmaceutica. Tale costo non corrisponde al prezzo al pubblico di amoxicillina, in quanto le farmacie applicano al SSN uno sconto che varia in funzione del tipo di farmacia e del prezzo del farmaco; per ragioni pratiche è stato assunto uno sconto per il SSN pari a quello base del $4 \%$ circa.

Pertanto, l'uso di amoxicillina per OMA, sinusite e altre complicanze costa al Sistema Sanitario rispettivamente 2,60 euro a confezione moltiplicati per il numero di confezioni necessarie secondo lo schema terapeutico seguito (vedere appendice - Calcolo del costo di amoxicillina). 


\section{- Prospettiva della società}

Il costo diretto che grava sulla collettività in caso di complicanza (con risposta al trattamento di prima linea) comprende:

- il costo della visita pediatrica;

- il costo di amoxicillina (costo a carico del $\mathrm{SSN}+$ costo a carico del paziente);

- il costo del paracetamolo;

- il costo dei decongestionanti in caso di sinusite;

- il costo dei farmaci mucolitici ed espettoranti in caso di bronchite.

Costi diretti associati a OMA e a sinusite non rispondenti al trattamento di prima $\underline{\text { scelta }}$

Le linee guida $[15,16,32,33,41,46-49]$ suggeriscono il passaggio ad un trattamento antibiotico di seconda linea nel caso in cui trascorse 72 ore di terapia con amoxicillina, il pediatra non ravvisi nessun miglioramento del quadro clinico del paziente. Gli antibiotici raccomandati in questo caso dalla maggior parte delle linee guida sono amoxicillina/acido clavulanico e cefuroxima.

\section{- Prospettiva del paziente}

In caso di mancata risoluzione della complicanza il paziente deve sostenere, oltre ai costi annoverati fin qui, la spesa per l'acquisto dei farmaci antibatterici di seconda linea che è stata stimata in base alla media dei costi relativi alle due alternative farmacologiche proposte dalle linee guida (costo del ticket moltiplicato per le confezioni richieste dallo schema posologico) (vedere appendice - Calcolo del costo per terapia antibiotica di seconda linea).

\section{- Prospettiva del SSN}

Il costo sostenuto dal SSN per ogni complicanza che non si risolve con il trattamento di prima linea corrisponde alla media fra i costi d'acquisto (al netto dello sconto) degli antibiotici di seconda scelta raccomandati dalle principali linee guida (vedere appendice - Calcolo del costo per terapia antibiotica di seconda linea).

Ai costi già elencati va sommato, in caso di non risposta al trattamento di prima linea, il costo di una seconda visita pediatrica (12,32 euro).

\section{- Prospettiva della società}

Il costo diretto per la società è costituito dall'insieme dei costi descritti per paziente e SSN.

Costi diretti associati a OMA e a sinusite non rispondenti al trattamento di $\underline{\text { seconda scelta }}$

$\mathrm{Nel}$ caso in cui sinusite o otite media persistano, le principali linee guida $[15,16,32,33]$ suggeriscono di consultare uno specialista in otorinolaringoiatria che possa valutare la gravità della complicanza, richiedere esami specifici al fine di mettere a punto una terapia speci- fica, prescrivere una terapia antibiotica di terza linea $\mathrm{o}$, in caso di complicanze gravi, richiedere l'ospedalizzazione.

Il costo associato all'ulteriore aggravamento del quadro clinico (o comunque alla "non risoluzione" della patologia con la terapia antibiotica di seconda scelta) è stato valutato in base al costo della terapia antibiotica di terza linea raccomandata dalle linee guida e alle tariffe DRG (Diagnosis Related Group) applicate in caso di ricovero, tenendo conto delle probabilità associate a tali eventi.

Il ricovero può essere richiesto per agevolare la somministrazione di una terapia antibiotica intravenosa oppure può risultare indispensabile per la comparsa di complicanze pericolose per la vita. Tali complicanze con i relativi DRG sono riassunte in Tabella III.

Il costo di ospedalizzazione associato a OMA e a sinusite acuta non rispondenti al trattamento di seconda linea è stato stimato in base alla media fra queste tariffe pesate per la loro frequenza relativa; i dati necessari sono stati tratti dalle banche dati statistiche sui ricoveri ospedalieri del Ministero della Salute [52].

Abbiamo assunto che nei casi di OMA e sinusite (non rispondenti a terapia di seconda linea) in cui non si ricorra al ricovero in ospedale, l'otorinolaringoiatra prescriva una terapia antibiotica di terza scelta; tale terapia secondo le linee guida dovrebbe essere costituita da ceftriaxone o clindamicina nel trattamento dell'otite media e clindamicina o cefixima in caso di sinusite.

Il costo da imputare ad ogni episodio è stato calcolato in base alle media dei costi d'acquisto di questi farmaci.

\section{- Prospettiva del paziente}

Le spese che il nucleo familiare deve sostenere sono legate al costo della visita specialistica e ai costi di farmacia.

\begin{tabular}{|c|c|c|c|}
\hline DRG & Denominazione & $\begin{array}{r}\text { Diagnosi o } \\
\text { intervento }\end{array}$ & $\begin{array}{c}\text { Tariffa } \\
\text { (in euro) }\end{array}$ \\
\hline $070 \mathrm{M}$ & $\begin{array}{l}\text { Otite media e } \\
\text { infezioni alte vie } \\
\text { respiratorie, età }<18\end{array}$ & $\begin{array}{l}\text { Otite media suppurativa e non } \\
\text { specificata } \\
\text { Otite media non suppurativa e } \\
\text { disturbi della tromba di Eustachio } \\
\text { Sinusite acuta } \\
\text { Mastoidite }\end{array}$ & $1.090,26$ \\
\hline $020 \mathrm{M}$ & $\begin{array}{l}\text { Infezioni del sistema } \\
\text { nervoso eccetto } \\
\text { meningite virale }\end{array}$ & $\begin{array}{l}\text { Meningite batterica } \\
\text { Ascesso cerebellare, cerebrale, } \\
\text { epidurale, extradurale }\end{array}$ & $4.714,65$ \\
\hline $003 \mathrm{C}$ & Craniotomia età $<18$ & $\begin{array}{l}\text { Drenaggio di igroma intracranico, } \\
\text { di ascesso subaracnoideo, di } \\
\text { empiema sottodurale }\end{array}$ & $8.793,34$ \\
\hline 062 C & $\begin{array}{l}\text { Miringotomia con } \\
\text { inserzione di tubo, } \\
\text { età }<18\end{array}$ & $\begin{array}{l}\text { Miringotomia con inserzione di } \\
\text { tubo }\end{array}$ & $1.321,23$ \\
\hline $044 \mathrm{M}$ & $\begin{array}{l}\text { Infezioni acute } \\
\text { maggiori dell'occhio }\end{array}$ & Cellulite e ascesso orbitario & $2.131,20$ \\
\hline
\end{tabular}

$\mathrm{M}=$ medici, $\mathrm{C}=$ chirurgici

Tabella III

Principali cause di ricovero in bambini con OMA o sinusite acuta e loro tariffe DRG 
Secondo la delibera della Giunta Regionale del Piemonte N'73-13176 la quota di partecipazione del paziente alla spesa sanitaria relativa ad una visita laringoiatrica è pari a 18,60 euro [53]; per l'acquisto degli antibiotici di terza scelta è a carico del paziente il costo del ticket (vedere appendice - Calcolo del costo per terapia antibiotica di terza linea).

\section{- Prospettiva del SSN}

Secondo la sopracitata delibera regionale, per prestazioni di assistenza specialistica ambulatoriale, il Sistema Sanitario riconosce alle strutture accreditate una somma pari a 30,00 euro.

Sono inoltre a carico del Sistema Sanitario le spese relative all'ospedalizzazione (vedere appendice - Calcolo del costo di ospedalizzazione) e all'acquisto degli antibiotici al netto del ticket pagato dal paziente (vedere appendice - Calcolo del costo per terapia antibiotica di terza linea).

\section{- Prospettiva della società}

Sono a carico della collettività i costi di farmacia, delle visite e di ospedalizzazione nella loro globalità.

\section{Analisi di sensibilità}

Per verificare la validità dei risultati ottenuti si è svolta un'analisi di sensibilità; in tale analisi si misura l'impatto indotto da una serie di variazioni delle ipotesi di partenza, cruciali o più incerte, sui risultati ottenuti; qualora questi ultimi non subiscano importanti cambiamenti, possono considerarsi attendibili [54].

Nel caso in questione l'analisi di sensibilità è stata svolta facendo variare i parametri più frequentemente soggetti a variazioni o più esposti ad errori di valutazione, e sono state analizzate le conseguenze che queste variazioni hanno per il costo marginale.

\section{Analisi di soglia}

Un particolare tipo di analisi di sensibilità (threshold analysis) permette di calcolare, in riferimento ad un determinato parametro, il valore per cui le alternative confrontate diventano indifferenti sotto il profilo economico, cioè, nel nostro caso, la condizione per cui il trattamento preventivo con OM- 85 smetterebbe di essere conveniente. Tale soglia è stata calcolata in riferimento a due parametri fondamentali per la nostra analisi: l'efficacia del vaccino (espressa come rischio relativo - RR) e il costo di un episodio.

\section{RISULTATI}

\section{Efficacia marginale della prevenzione}

Il numero di infezioni per paziente è stato calcolato pesando il numero medio di infezioni osservato nei trial selezionati (Tabella I) per la numerosità del campione analizzato.

In riferimento ad un periodo di osservazione di 6 mesi sono stati ottenuti i seguenti risultati:

- il numero medio di infezioni nel gruppo trattato con OM-85 è stato pari a 1,70 ;

- il numero medio di infezioni nel gruppo controllo è stato pari a 3,30;

- il numero medio pesato di infezioni prevenute da OM-85 è quindi risultato di 1,60 (Rischio Relativo =0,515). Questo dato rappresenta l'efficacia della prevenzione.

In riferimento ad un periodo di osservazione di 12 mesi sono stati utilizzati i dati del trial condotto da Gutiérrez-Tarango, in quanto unico trial selezionato:

- il numero medio di infezioni nel gruppo trattato è risultato pari a 5,04;

- il numero medio di infezioni nel gruppo controllo è risultato pari a 8 ;

- il numero medio di infezioni prevenute da OM-85 è quindi pari a 2,96 (Rischio Relativo $=0,63)$.

\section{Costo marginale della prevenzione}

Il costo marginale della prevenzione corrisponde al costo diretto del vaccino al quale è

\section{Tabella IV}

Costo sostenuto dal nucleo familiare per periodo di osservazione (in euro)

\begin{tabular}{|c|c|c|c|c|c|c|}
\hline \multirow{2}{*}{ Scenario } & \multicolumn{2}{|c|}{$\begin{array}{l}\text { Paziente } \\
\text { vaccinato }\end{array}$} & \multicolumn{2}{|c|}{$\begin{array}{l}\text { Paziente non } \\
\text { vaccinato }\end{array}$} & \multirow{2}{*}{$\begin{array}{c}\text { Costo } \\
\text { marginale } \\
\text { prevenzione }\end{array}$} & \multirow{2}{*}{$\begin{array}{c}\text { Costo/ } \\
\text { Episodio } \\
\text { evitato }\end{array}$} \\
\hline & $\begin{array}{c}\text { Costo } \\
\text { tot }\end{array}$ & $\begin{array}{c}\mathbf{N}^{\prime} \\
\text { episodi }\end{array}$ & $\begin{array}{c}\text { Costo } \\
\text { tot }\end{array}$ & $\begin{array}{c}\mathbf{N}^{\prime} \\
\text { episodi }\end{array}$ & & \\
\hline $\begin{array}{l}\text { Scenario 1) } \\
\text { Periodo considerato: } 6 \text { mesi } \\
1 \text { ciclo di vaccino }\end{array}$ & 150,85 & 1,70 & 258,27 & 3,30 & $-107,42$ & Dominanza \\
\hline $\begin{array}{l}\text { Scenario 2) } \\
\text { Variazione proporzione URTI } \\
\text { NC/URTI CC }\end{array}$ & 150,43 & 1,70 & 258,27 & 3,30 & $-107,84$ & Dominanza \\
\hline $\begin{array}{l}\text { Scenario 3) } \\
\text { No ticket farmaceutici }\end{array}$ & 148,71 & 1,70 & 254,27 & 3,30 & $-105,56$ & Dominanza \\
\hline $\begin{array}{l}\text { Scenario 4) } \\
\text { No costi indiretti }\end{array}$ & 30,81 & 1,70 & 25,25 & 3,30 & 5,56 & 3,29 \\
\hline $\begin{array}{l}\text { Scenario 5) } \\
\text { Periodo considerato: } 12 \text { mesi } \\
2 \text { cicli di vaccino }\end{array}$ & 430,10 & 5,04 & 626,18 & 8 & $-196,08$ & Dominanza \\
\hline
\end{tabular}




\begin{tabular}{|c|c|c|c|c|c|c|}
\hline \multirow{2}{*}{ Scenario } & \multicolumn{2}{|c|}{$\begin{array}{c}\text { Paziente } \\
\text { vaccinato }\end{array}$} & \multicolumn{2}{|c|}{$\begin{array}{l}\text { Paziente non } \\
\text { vaccinato }\end{array}$} & \multirow{2}{*}{$\begin{array}{c}\text { Costo } \\
\text { marginale } \\
\text { prevenzione }\end{array}$} & \multirow{2}{*}{$\begin{array}{l}\text { Costo/ } \\
\text { Episodio } \\
\text { evitato }\end{array}$} \\
\hline & $\begin{array}{c}\text { Costo } \\
\text { tot }\end{array}$ & $\begin{array}{c}\mathbf{N}^{\prime} \\
\text { episodi }\end{array}$ & $\begin{array}{c}\text { Costo } \\
\text { tot }\end{array}$ & $\begin{array}{c}\mathbf{N}^{\prime} \\
\text { episodi }\end{array}$ & & \\
\hline $\begin{array}{l}\text { Scenario 1) } \\
\text { Periodo considerato: } 6 \text { mesi } \\
1 \text { ciclo di vaccino }\end{array}$ & 51,54 & 1,70 & 100,06 & 3,30 & - 48,52 & Dominanza \\
\hline $\begin{array}{l}\text { Scenario 2) } \\
\text { Variazione proporzione URTI } \\
\text { NC/URTI CC }\end{array}$ & 36,89 & 1,70 & 100,06 & 3,30 & $-63,17$ & Dominanza \\
\hline $\begin{array}{l}\text { Scenario 3) } \\
\text { No ticket farmaceutici }\end{array}$ & 53,68 & 1,70 & 104,20 & 3,30 & $-50,52$ & Dominanza \\
\hline $\begin{array}{l}\text { Scenario 4) } \\
\text { No visite pediatriche }\end{array}$ & 33,26 & 1,70 & 64,57 & 3,30 & $-31,31$ & Dominanza \\
\hline $\begin{array}{l}\text { Scenario 5) } \\
\text { Periodo considerato: } 12 \text { mesi } \\
2 \text { cicli di vaccino }\end{array}$ & 93,53 & 5,04 & 148,46 & 8 & $-54,93$ & Dominanza \\
\hline
\end{tabular}

\begin{tabular}{|c|c|c|c|c|c|c|}
\hline \multirow{2}{*}{ Scenario } & \multicolumn{2}{|c|}{$\begin{array}{c}\text { Paziente } \\
\text { vaccinato }\end{array}$} & \multicolumn{2}{|c|}{$\begin{array}{l}\text { Paziente non } \\
\text { vaccinato }\end{array}$} & \multirow{2}{*}{$\begin{array}{c}\text { Costo } \\
\text { marginale } \\
\text { prevenzione }\end{array}$} & \multirow{2}{*}{$\begin{array}{l}\text { Costo/ } \\
\text { Episodio } \\
\text { evitato }\end{array}$} \\
\hline & $\begin{array}{c}\text { Costo } \\
\text { tot }\end{array}$ & $\begin{array}{c}\mathbf{N}^{\prime} \\
\text { episodi }\end{array}$ & $\begin{array}{c}\text { Costo } \\
\text { tot }\end{array}$ & $\begin{array}{c}\mathbf{N}^{\prime} \\
\text { episodi }\end{array}$ & & \\
\hline $\begin{array}{l}\text { Scenario 1) } \\
\text { Periodo considerato: } 6 \text { mesi } \\
1 \text { ciclo di vaccino }\end{array}$ & 282,42 & 1,70 & 513,68 & 3,30 & $-231,26$ & Dominanza \\
\hline $\begin{array}{l}\text { Scenario 2) } \\
\text { Variazione proporzione URTI } \\
\text { NC/URTI CC }\end{array}$ & 267,35 & 1,70 & 513,68 & 3,30 & $-246,33$ & Dominanza \\
\hline $\begin{array}{l}\text { Scenario 3) } \\
\text { No visite pediatriche }\end{array}$ & 264,14 & 1,70 & 478,19 & 3,30 & $-214,05$ & Dominanza \\
\hline $\begin{array}{l}\text { Scenario 4) } \\
\text { Periodo considerato: } 12 \text { mesi } \\
2 \text { cicli di vaccino }\end{array}$ & 814,81 & 5,04 & $\begin{array}{c}1.236,- \\
83\end{array}$ & 8 & $-422,02$ & Dominanza \\
\hline
\end{tabular}

sottratto il costo risparmiato in seguito alla riduzione del numero di eventi da trattare. In altre parole esso è dato dalla differenza fra il costo per paziente vaccinato (quindi comprensivo di costo diretto del vaccino) e il costo per paziente non vaccinato:

\section{Costo PZ vaccin./periodo di osservazione}

Costo PZ non vaccin./periodo di osservazione

Riportiamo in Tabella IV, V, VI il costo sostenuto per paziente trattato con OM-85, per paziente trattato con placebo e il costo marginale di prevenzione nei vari scenari presi in esame e secondo le tre diverse prospettive considerate. Il segno "meno" davanti ai valori corrispondenti al costo marginale di prevenzione indica che tale costo nel caso in esame è invece un risparmio (per paziente per periodo considerato).

\section{Rapporto marginale di costo/efficacia}

Il valore di costo/efficacia marginale corrisponde al costo per episodio evitato ed è calcolato dividendo il costo marginale della prevenzione (costo $\mathrm{pz}$ vaccinato - costo pz non vaccinato) per il numero medio di episodi evitati.

Nello scenario 4 della Tabella IV, in cui non sono stati considerati i costi indiretti, il costo/ paziente trattato con $\mathrm{OM}-85$ è risultato pari a
30,81 euro e il costo/paziente trattato con placebo pari a 25,25 euro. In tal caso dividendo la differenza $(5,56$ euro) per il numero di episodi evitati $(1,60)$ si ottiene un valore di costo/efficacia marginale di 3,29 euro.

In tutti gli altri casi, il vaccino ha dimostrato un'efficacia maggiore di placebo e un costo complessivo minore: condizione di dominanza farmacoeconomica.

\section{Analisi di sensibilità}

La Tabella VII riporta i principali parametri che sono stati fatti variare, unitamente ai loro valori basali, alle variazioni che sono state adottate e alla prospettiva assunta nel corso dell'analisi di sensibilità.

L'analisi di sensibilità è stata svolta solo sullo scenario base (scenario 1) e sullo scenario a 12 mesi.

La Figura 4 rappresenta i valori di efficacia del vaccino (RR) e di costo/episodio per cui la differenza costo/periodo tra un paziente vaccinato e uno non vaccinato si annulla. La prospettiva assunta è quella del paziente.

\section{DISCUSSIONE DEI RISULTATI}

Secondo il modello da noi implementato, un ciclo di trattamento preventivo con OM-85

\section{Analisi di soglia}

\section{Tabella V}

Costo sostenuto dal SSN per periodo di osservazione (in euro)

\section{Tabella VI}

Costo sostenuto dalla società per periodo di osservazione (in euro) 


\section{Tabella VII}

Ipotesi testate nel corso dell'analisi di sensibilità
Figura 2

Analisi di sensibilità: A. Ipotesi 3 - 6 mesi (prospettiva paziente); B. Ipotesi 3 - 12 mesi

Figura 3

Analisi di sensibilità A. Ipotesi 4 - 6 mesi (prospettiva SSN); B. Ipotesi 4 - 12 mesi (prospettiva SSN)

\begin{tabular}{|c|c|c|c|}
\hline Parametro & Valore basale & Ipotesi testate & $\begin{array}{c}\text { Prospettiva } \\
\text { assunta }\end{array}$ \\
\hline \multirow[t]{2}{*}{ Rischio Relativo } & RR 6 mesi $=0,515$ & $\mathrm{I}_{1}$ ) Variazione RR nell'intervallo 0,515 $\pm 20 \%$ & Paziente \\
\hline & RR 12 mesi $=0,63$ & Variazione RR nellintervallo $0,63 \pm 20 \%$ & \\
\hline $\begin{array}{l}\text { Probabilità URTI } \\
\text { NC }\end{array}$ & $p=0,69$ & $\begin{array}{l}\left.\mathrm{I}_{2}\right) \text { Probabilità fatta variare da } 0,57 \text { a } 0,89 \text {, } \\
\text { valori minimo e massimo reperiti in } \\
\text { letteratura [11-13] }\end{array}$ & $\begin{array}{l}\text { Paziente, } \\
\text { SSN }\end{array}$ \\
\hline $\begin{array}{l}\text { Giorni di lavoro } \\
\text { persi }\end{array}$ & 3,5 & $\begin{array}{l}\mathrm{I}_{3} \text { ) II numero di giorni persi è stato fatto } \\
\text { variare da un minimo di } 1 \text { giorno ad un } \\
\text { massimo di } 7 \text { giorni, durata media di un } \\
\text { episodio di URTI [11] }\end{array}$ & Paziente \\
\hline $\begin{array}{l}\text { Costo } \\
\text { ospedalizzazione }\end{array}$ & $\begin{array}{l}\text { Per OMA complicata }= \\
1.999,04 \text { euro } \\
\text { Per sinusite complicata }= \\
2.230,25 \text { euro }\end{array}$ & $\begin{array}{l}\mathrm{I}_{4} \text { ) il costo di ospedalizzazione si è fatto } \\
\text { variare fra } 1.090,26 \text { e } 8.793,34 \text { euro, valori } \\
\text { entro cui variano le tariffe DRG riportate in } \\
\text { Tabella III }\end{array}$ & SSN \\
\hline
\end{tabular}

A.

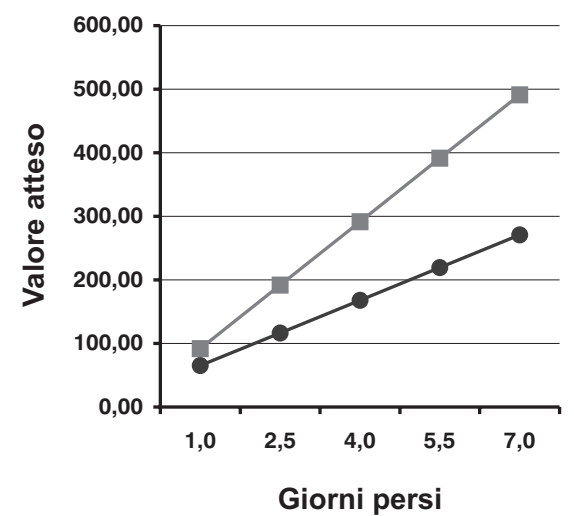

B.

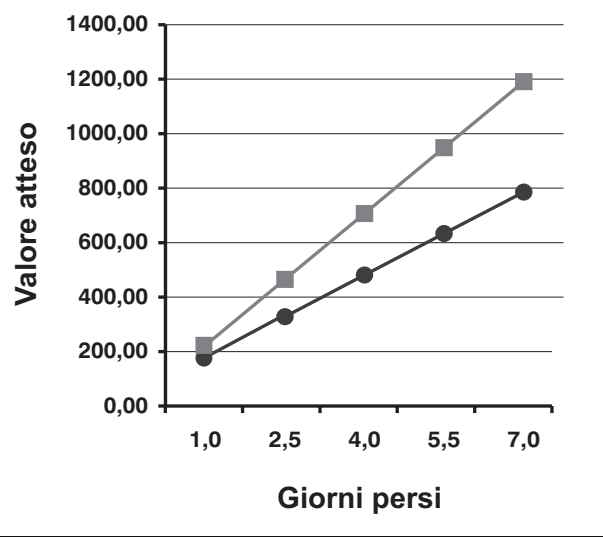

A.

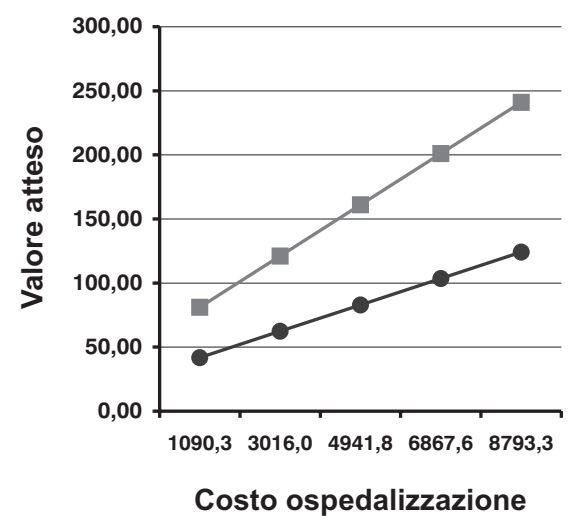

B.

$\longrightarrow$ Vaccino

- No vaccino

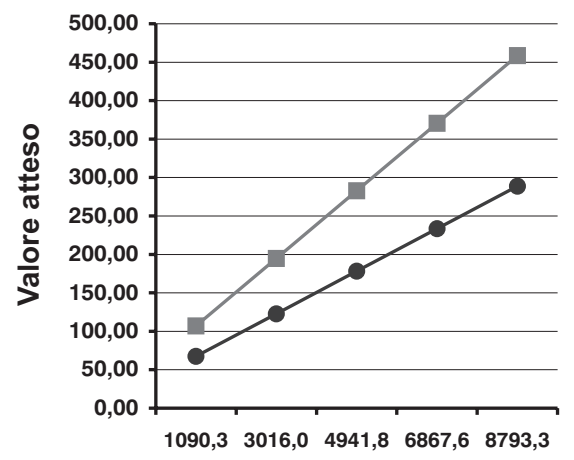

Costo ospedalizzazione comporta un risparmio per il paziente di 107,42 euro in sei mesi (scenario 1 - Tabella IV). Tale risparmio è dovuto al fatto che il vaccino, secondo i trial presi in esame, è in grado di ridurre del 48,5\% la comparsa di infezioni alle vie aere superiori nei pazienti a rischio.

I costi che il nucleo familiare deve sostenere in caso di URTI sono molteplici: costi farmacologici associati all'utilizzo di farmaci antipiretici, analgesici, antibiotici, il costo delle visite specialistiche, $\mathrm{i}$ costi indiretti correlati alla perdita di giorni di lavoro, ecc. La riduzio- ne complessiva di tali spese, indotta dal minor numero di URTI/periodo, è in grado, come dimostrato da tale risultato, di compensare ampiamente il prezzo d'acquisto del vaccino.

Tale vantaggio economico è mantenuto anche considerando un periodo di osservazione di 12 mesi con due cicli di vaccino (risparmio di 196,08 euro/12 mesi).

Dal momento che in Italia non vi è uniformità tra regioni per ciò che riguarda la partecipazione del paziente alla spesa farmaceutica (pagamento del ticket sui farmaci rimborsabili), 
l'analisi dei costi è stata condotta considerando due diversi scenari: l'applicazione di un ticket di 2,00 euro per ogni confezione di farmaco di fascia A acquistata (scenario 1 - Tabella IV) e la completa rimborsabilità da parte del SSN (scenario 3 - Tabella IV). Il risparmio calcolato in base a quest'ultimo scenario è di poco dissimile rispetto allo scenario base (circa di 2,00 euro inferiore); la ragione è molto semplice: circa il $70 \%$ dei casi di URTI si risolve senza complicazioni batteriche e, di conseguenza, senza richiedere la somministrazione di antibatterici o di altri farmaci di fascia A. Inoltre l'eliminazione del ticket non abbatte in maniera così rilevante il costo globale dell'episodio da invertire la tendenza al risparmio indotta dalla prevenzione.

Nel secondo scenario della Tabella IV è stato assunto che la somministrazione del vaccino, oltre ad influire sul numero di episodi/periodo, diminuisse anche la frequenza di comparsa di complicanze. Dai risultati di quest'analisi ci si sarebbe aspettato un risparmio da prevenzione molto più elevato di quello risultante dallo scenario base, a causa del fatto che la comparsa di complicanze induce sempre un elevato aumento dei costi: maggiori costi di farmacia, incremento del numero di visite, aumento dei giorni lavorativi persi. Il motivo per cui il nostro modello non rileva questa sostanziosa differenza è da ricercare proprio in quest'ultimo parametro; per ragioni pratiche, infatti, per stabilire la probabilità con cui un genitore si assenta dal lavoro a causa di problemi di salute del figlio e il numero di giorni di lavoro persi ci si è riferiti a valori medi reperiti in letteratura; tali valori, chiaramente, non tengono conto del fatto che in caso di infezione con complicanze, una percentuale maggiore di genitori si assenterà dal lavoro (aumenta la necessità di cure, di visite mediche, ecc.). Secondo lo stesso ragionamento, un episodio complicato richiede un maggior numero di giorni per risolversi e, necessariamente, il paziente perderà più giorni di scuola rispetto ad un comune raffreddore $\mathrm{e}$, probabilmente, questo si rifletterà sul numero di giorni in cui i genitori non potranno recarsi al lavoro. Tenendo conto di queste considerazioni è facile intuire come una riduzione del rischio di complicanze possa indurre un risparmio ben più elevato rispetto a quello calcolato dal nostro modello.

Considerata la grande variabilità dei costi indiretti e le conseguenti difficoltà nel trovare un valore medio che rispecchi il più possibile la realtà, il costo marginale di prevenzione è stato calcolato anche tenendo conto solo dei costi diretti (scenario 4 - Tabella IV).

In questo caso ogni famiglia spenderebbe 3,29 euro per ogni episodio evitato $(5,56$ euro a semestre). Questo costo è trascurabile non solo per l'esiguità della cifra, ma soprattutto perché, se da un punto di vista puramente clinico

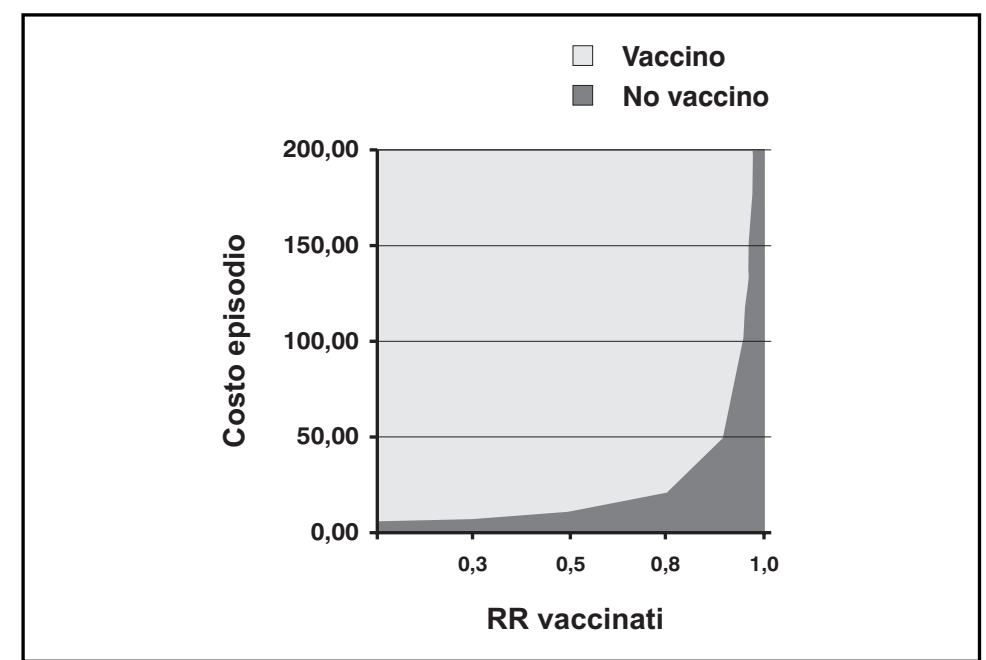

Figura 4

Analisi di soglia

riuscire ad evitare un episodio di URTI può sembrare un risultato marginale, per un paziente pediatrico e per chi se ne prende cura può invece rappresentare un beneficio importante.

Occorre anche tenere presente che alcuni costi collegati essenzialmente alla giovane età dei pazienti, come ad esempio il costo del trasporto dal pediatra, il costo di baby-sitter o la perdita di giorni di scuola, per ragioni pratiche non sono stati considerati nel modello; tali costi hanno spesso un valore elevato e bilanciano ampiamente tale cifra.

Secondo la prospettiva del SSN, la prevenzione con OM-85 rappresenta una strategia conveniente (Tabella V) in tutti gli scenari da noi considerati.

Diminuendo il numero degli episodi URTI, si riducono proporzionalmente i costi a carico del Sistema Sanitario ad essi associati (visite, farmaci di fascia A, ospedalizzazione); a fronte di questo risparmio il SSN non deve sostenere nessun costo aggiuntivo visto che la spesa per l'acquisto del vaccino è a carico del paziente. Secondo i risultati ottenuti, il risparmio conseguito permetterebbe al SSN di farsi carico del costo di tale farmaco: anche in tal caso il suo utilizzo risulterebbe conveniente.

Nell'ipotesi che il vaccino abbia effetto anche sul rischio di comparsa di complicanze (scenario 2 - Tabella V), il SSN otterrebbe un risparmio circa del $30 \%$ in più rispetto a quello risultante dallo scenario base. Questo è essenzialmente dovuto al fatto che le complicanze inducono un consumo di risorse per il SSN molto superiore all'episodio semplice il quale, secondo le principali linee guida, non necessita per la sua risoluzione di consulti specialistici, di farmaci antibatterici e di ospedalizzazione. Vi è poi una considerevole percentuale di casi ( $25 \%$ circa) in cui il paziente con URTI non complicato, non è visitato neppure dal pediatra di famiglia: in tali casi il costo per il SSN è ancora minore. 
Anche nel caso in cui le visite pediatriche, avendo un costo teoricamente nullo per le ragioni spiegate in precedenza, non fossero inserite nel computo dei costi (scenario 4 - Tabella V), il risultato dell'analisi risulterebbe sempre a favore della terapia preventiva.

Nell'ottica della collettività valgono tutte le osservazioni fatte fin qui; tutti gli scenari ideati hanno dimostrato la convenienza della prevenzione con OM-85.

Naturalmente al dato puro che indica il risparmio ottenibile dalla collettività grazie alla riduzione del numero di episodi (231,26 euro per lo scenario base) va aggiunto, in questa prospettiva più ancora che nelle altre, il beneficio generale indotto dalla prevenzione di URTI. Un minor numero di infezioni delle vie aeree significa, infatti, meno pazienti che perdono giorni di scuola, minor rischio di contagio, minore perdita di produzione per i genitori e per i datori di lavoro, minor consumo di risorse sanitarie e conseguente attesa per riceverle.

\section{Analisi di sensibilità e di soglia}

L'analisi di sensibilità ha dimostrato che, all'interno dei range di variazione dei parametri selezionati, non si verifica alcuna condizione per cui il costo di un paziente a rischio trattato preventivamente sia maggiore del costo di un paziente non trattato.

L'entità del risparmio varia a seconda delle ipotesi testate.

Facendo variare l'efficacia del vaccino abbiamo notato che anche riducendola del $20 \%$ (RR $=0,61$ per 6 mesi e $R R=0,75$ per 12 mesi) il risparmio per il paziente rimane ancora elevato $(80,00$ euro circa per sei mesi e 120,00 per 12 mesi).

Per quanto riguarda la probabilità che un URTI si complichi, si è osservato che, assumendo come valori minimo e massimo quelli reperiti in letteratura, l'andamento dei costi riflette le considerazioni fatte in precedenza: il vantaggio per il paziente rimane, da un punto di vista economico e secondo il modello matematico, pressoché costante, mentre per il SSN l'abbattimento di tale rischio permette un consumo di risorse per episodio molto più limitato; la strategia preventiva rimane tuttavia conveniente anche qualora il tasso di complicanze fosse ridotto all' $11 \%$.

I giorni di lavoro persi, come già detto, rappresentano un costo molto variabile, la cui determinazione richiede inevitabilmente l'assunzione di ipotesi generiche e spesso imprecise. Per tale ragione si è fatto variare il parametro "giorni di lavoro persi" da uno a sette (durata media di un URTI). Come si può osservare dalla Figura 2, la prevenzione con OM-85 risulta dominante anche qualora fosse perso un solo giorno di lavoro; questo dato deve essere valutato nel giusto contesto: il nostro modello assume l'ipotesi che i genitori (la madre per esattezza) si assentino dal lavoro solo nel $34,2 \%$ dei casi, assunzione conservativa se si considera che alcune stime riportano una percentuale del $55,4 \%$.

Come mostrato in Figura 3, facendo variare il costo di ospedalizzazione, varia l'entità del risparmio per il SSN; tale variazione tuttavia è limitata e in nessun punto del range adottato, la strategia preventiva perde la sua convenienza.

Infine, l'analisi di soglia ci ha permesso di valutare per quali valori di efficacia e di costo/ episodio, il trattamento con OM-85 diventa economicamente meno vantaggioso della nonprevenzione.

Come si evince dal grafico, ad un valore di efficacia pari a quello utilizzato nel nostro modello $(R R=0,515)$ il vaccino resta conveniente fino a che i costi per episodio non scendano sotto il valore di circa 10,00 euro.

Inversamente, mantenendo fisso il costo di un episodio, calcolato nel modello (circa 75,00 euro), il vaccino resta conveniente finché in grado di ridurre almeno del $7 \%$ il rischio di sviluppare un'infezione $(\mathrm{RR}=0,93)$.

\section{Limiti dell'analisi}

Il primo limite dell'analisi è dovuto al fatto che la qualità di un modello farmacoeconomico dipende fondamentalmente dalla qualità degli studi da cui derivano i dati di efficacia. In questo caso l'effetto di OM-85 è stato analizzato da più studi, i quali, sebbene con uguali criteri d'inclusione e principali outcomes, derivano da contesti diversi e con pazienti di età media e condizioni di vita leggermente differenti; due di essi sono svolti poi su un campione non molto numeroso.

Un altro limite è rappresentato dalla difficoltà nell'ottenere dati certi e uniformi. I costi diretti di un episodio URTI sono stati calcolati in base allo schema terapeutico raccomandato nelle principali linee guida; questo metodo inevitabilmente comporta l'ottenimento di valori di costo leggermente diversi da quelli reali, in quanto spesso la pratica clinica, basata sostanzialmente sull'esperienza del medico o del pediatra, non è perfettamente allineata alle raccomandazioni.

Altri problemi di disomogeneità derivano dal fatto che le regioni italiane godono di un'autonomia che permette loro di decidere l'importo delle tariffe DRG e delle quote di partecipazione alla spesa sanitaria (ticket su visite ed esami) e alla spesa farmaceutica (ticket sui farmaci). Per tali motivi si possono ottenere risultati diversi a seconda della fonte utilizzata per stimare i costi dei servizi: le differenze, comunque, sono solitamente lievi e in questo lavoro si è cercato di usare come base di calcolo prezzari di regioni con tariffe intermedie.

\section{CONCLUSIONI}

Il nostro intento è stato quello di valutare la costo/efficacia di OM-85 nel prevenire la 
comparsa di infezioni alle alte vie respiratorie in bambini a rischio.

A tal fine abbiamo combinato i dati clinici ed epidemiologici presenti in letteratura con $i$ dati economici riferiti allo stato italiano, ricavati da banche dati statistiche, tariffari regionali, prontuari farmaceutici e studi farmacoeconomici precedenti.

Con questi dati è stato implementato un albero decisionale al fine di calcolare, assumendo ipotesi ragionevoli, il costo diretto e indiretto associato ad un paziente pediatrico con URTI ricorrenti e di confrontarlo con il costo per lo stesso periodo associato ad un paziente trattato preventivamente con il lisato batterico.

Dai dati di efficacia del farmaco, forniti da studi controllati e randomizzati, è emerso che un ciclo di immunostimolante OM-85 evita ai bambini a rischio in media 1,60 episodi in 6 mesi.

Tale risultato comporta una diminuzione dei costi diretti e indiretti a carico del paziente e della società che bilancia ampiamente il costo del vaccino, che equivale a 17,80 euro per ciclo di trattamento.

Il risparmio per il paziente è stato calcolato essere pari a 107,42 euro a semestre; per la società tale valore si aggira intorno ai 231,26 euro.

Sono stati ipotizzati diversi scenari per avvicinarsi il più possibile alle diverse realtà regionali italiane e per assecondare la disomogeneità che spesso caratterizza i dati reperiti in letteratura. I vari scenari hanno comunque confermato la convenienza della strategia profilattica.

Anche per il SSN, la somministrazione di OM-85 e la conseguente riduzione del numero di infezioni comporta un risparmio notevole, dovuto alla riduzione delle risorse consumate per la cura di tali eventi e, soprattutto, delle potenziali complicanze. Il risparmio per paziente è risultato pari a 48,52 euro in sei mesi (scenario base).

Le analisi di sensibilità hanno confermato la robustezza di tali risultati.

Nonostante i naturali limiti metodologici, questo lavoro dimostra che l'immunoterapia aspecifica realizzata con OM- 85 costituisce un intervento ragionevole in bambini a rischio e che dovrebbe essere adottata in aggiunta ad altre misure raccomandate, come ad esempio la correzione di fattori di rischio noti.

\section{APPENDICE}

Calcolo dei costi indiretti medi per episodio

12,29 euro $\times 8 \mathrm{~h}=98,32$ euro retribuzione giornaliera lorda

98,32 euro $\times 3,5 \mathrm{gg}=344,12$ euro costo indiretto sostenuto dalla società

$98,32-(98,32 \times 40 \%)=58.99$ euro retribuzione giornaliera netta

58,99 euro $\times 3,5 \mathrm{gg}=206,46$ euro costo indiretto sostenuto dal nucleo familiare

$\mathrm{P}=0,342$

Calcolo del costo farmaci sintomatici

Paracetamolo: 2,50 per episodio

Mucolitici/espettoranti: 6,50 euro

$\mathrm{P}=0,5$ per "altre complicanze"

Decongestionanti: 7,00 euro

$\mathrm{P}=0,65$ per URTI NN comp

$\mathrm{P}=1$ per sinusite 


\section{Calcolo del costo di amoxicillina \\ OMA risp 1' linea}

80 - $90 \mathrm{mg} / \mathrm{kg} /$ die per $7 \mathrm{gg}: 2$ conf. $12 \mathrm{cpr}$ da $1 \mathrm{~g}(1$ conf. $=4,70$ euro $)$

$2 \times 2,00$ euro $=4,00$ euro (ticket) a carico del paziente per episodio

$2 \times(2,70-$ sconto $4 \%)=5,20$ euro a carico del SSN per episodio

\section{Sinusite risp 1 ' linea}

$40-45 \mathrm{mg} / \mathrm{kg} / \mathrm{die}$ per $10 \mathrm{gg}$ : $1 \mathrm{conf} .12 \mathrm{cpr}$ da $1 \mathrm{~g}(1 \mathrm{conf} .=4,70$ euro $)$

2,00 euro (ticket) a carico del paziente per episodio

2,70 - sconto $4 \%=2,60$ euro a carico del SSN per episodio

\section{Altre complicanze}

$50 \mathrm{mg} / \mathrm{kg} /$ die per $7 \mathrm{gg}: 1$ conf. $12 \mathrm{cpr}$ da $1 \mathrm{~g}(1$ conf. $=4,70$ euro $)$

2,00 euro (ticket) a carico del paziente per episodio

2,70 - sconto $4 \%=2,60$ euro a carico del SSN per episodio

$\mathrm{P}=0,5$

\section{Calcolo del costo per terapia antibiotica di seconda linea}

\section{OMA e sinusite}

Amox/ac.clavulanico: 80-90 mg/kg/die per $10 \mathrm{gg}: 2$ conf. $12 \mathrm{cpr}$ da $1 \mathrm{~g}(1$ conf. = 18,26 euro $)$

$2 \times 2,00$ euro $=4,00$ euro (ticket) a carico del paziente

$2 \times(16,26$ - sconto $4 \%)=31,22$ euro a carico del SSN

Cefuroxima: $30 \mathrm{mg} / \mathrm{kg} /$ die per $10 \mathrm{gg}$ : 3 conf. 6 cpr da $500 \mathrm{mg}(1$ conf. $=14,65$ euro $)$

$3 \times 2,00$ euro $=6,00$ euro (ticket) a carico del paziente

$3 \times(12,65-$ sconto $4 \%)=36,30$ euro a carico del SSN

Media fra i due costi d'acquisto:

5,00 euro per il paziente

33,76 euro per il SSN

\section{Calcolo del costo per terapia antibiotica di terza linea}

\section{OMA}

Clindamicina fosfato IM: $20 \mathrm{mg} / \mathrm{kg} / \mathrm{die}$ per $10 \mathrm{gg}$ : 2 conf. $5 \mathrm{f}$ da $600 \mathrm{mg}(1$ conf. $=22,92$ euro $)$ $2 \times 2,00$ euro $=4,00$ euro (ticket) a carico del paziente

$2 \times(20,92$ - sconto $4 \%)=40,16$ euro a carico del ssn

Ceftriaxone im: $15-20 \mathrm{mg} / \mathrm{kg} /$ die per $10 \mathrm{gg}: 5$ conf. $1 \mathrm{f} \mathrm{da} 1 \mathrm{~g}$ ( 1 conf. $=6,6$ euro $)$

$5 \times 2,00$ euro $=10,00$ euro (ticket) a carico del paziente

$5 \times(4,6$ euro - sconto $4 \%)=22,08$ euro a carico del SSN

Media fra i due costi d'acquisto:

7,00 euro per il paziente

31,12 euro per il SSN

$\mathrm{P}=0,35$

\section{Sinusite}

Clindamicina fosfato IM: $20 \mathrm{mg} / \mathrm{kg} /$ die per $10 \mathrm{gg}$ : 2 conf. $5 \mathrm{f} \mathrm{da} 600 \mathrm{mg}(1 \mathrm{conf}$. $=22,92$ euro $)$

$2 \times 2,00$ euro $=4,00$ euro (ticket) a carico del paziente

$2 \times(20,92$ - sconto $4 \%)=40,16$ euro a carico del SSN

Cefixima: 6-8 mg/kg/die per $10 \mathrm{gg}$ : 1 flacone $100 \mathrm{ml}$ per os, $100 \mathrm{mg} / 5 \mathrm{ml}$ ( 1 conf. =21,86 euro)

2,00 euro (ticket) a carico del paziente

19,86 - sconto $4 \%=19,06$

Media fra i due costi d'acquisto:

3,00 euro (ticket) per il paziente

29,61 euro per il SSN

$\mathrm{P}=0,5$

\section{Costo di ospedalizzazione}

OMA non rispondente a trattamento di seconda linea $1.999,04$ euro (media pesata per la frequenza relativa) $\mathrm{P}=0,65$

Sinu non rispondente a trattamento di seconda linea 2.230,25 euro (media pesata per la frequenza relativa) $\mathrm{P}=0,5$ 


\section{BIBLIOGRAFIA}

1. De Baets: de Baets et al. IgG subclass specific antibody response in recurrent bronchitis. Arch Dis Child. 1991 Dec;66(12):1378-82

2. Mauel J. Stimulation of immunoprotective mechanisms by OM-85 BV. A review of results from in vivo and in vitro studies. Respiration. 1994;61 Suppl 1:8-15

3. Lusuardi $\mathrm{M}$ et al. Local airways immune modifications induced by oral bacterial extracts in chronic bronchitis. Chest. 1993 Jun;103(6):1783-91

4. Conférence de consensus en thérapeutique anti-infectieuse, 16/6/1996 (Lyon). Les infections ORL (texte long). Médecine et Maladies Infectieuses 1997; 27 spécial: 341-54

5. Maestroni GJ, Losa GA. Clinical and immunobiological effects of an orally administered bacterial extract. Int $\mathrm{J}$ Immunopharmacol. 1984;6(2):111-7.

6. Paupe J. Immunotherapy with an oral bacterial extract (OM-85 BV) for upper respiratory infections. Respiration. 1991;58(3-4):150-4.

7. Jara-Perez JV, Berber A. Primary prevention of acute respiratory tract infections in children using a bacterial immunostimulant: a double-masked, placebo-controlled clinical trial. Clin Ther. 2000 Jun;22(6):748-59.

8. Gutierrez-Tarango MD, Berber A. Safety and efficacy of two courses of OM-85 BV in the prevention of respiratory tract infections in children during 12 months. Chest. 2001 Jun;119(6):1742-8.

9. Van Cauwenberge $\mathrm{P}$ et al. Use of diagnostic clusters to assess the economic consequences of rhinopharyngitis in children in Italy and France during the winter. Clin Ther. 1999 Feb;21(2):404-21.

10. Wald ER. Sinusitis in children. N Engl J Med. 1992 Jan 30;326(5):319-23.

11. Wald ER, Guerra N, Byers C. Upper respiratory tract infections in young children: duration of and frequency of complications. Pediatrics. 1991 Feb;87(2):129-33.

12. Fahey T, Stocks N, Thomas T. Systematic review of the treatment of upper respiratory tract infection. Arch Dis Child. 1998 Sep;79(3):225-30.

13. Mazzeo F et al. Antibiotic drug prescription in respiratory tract infections: a pharmacoepidemiological survey among general practitioners in a region of Italy. J Chemother. 2000 Apr;12(2):153-9.

14. Dingle, J.H., G.F. Badger, and W.S.J. Jordan. 1964. Illness in the Home. A Study of 25,000 Illnesses in a Group of Cleveland Families. The Press of Western Reserve University, Cleveland. 347 pp.

15. Clinical practice guideline: management of sinusitis. American Academy of Pediatrics - Medical Specialty Society. 2001 Sep. 11

16. Cincinnati Children's Hospital Medical Center. Evidence based clinical practice guideline for children with acute bacterial sinusitis in children 1 to 18 years of age . Cincinnati $(\mathrm{OH})$ : Cincinnati Children's Hospital Medical Center; 2001 Apr 27.

17. Cohen R et al. Treatment failure in otitis media: an analysis. J Chemother. 1994 Sep;6 Suppl 4:17-22; discussion 23-4.

18. Le Saux N et al. A randomized, double-blind, placebo-controlled noninferiority trial of amoxicillin for clinically diagnosed acute otitis media in children 6 months to 5 years of age. CMAJ. 2005 Feb 1;172(3):335-41.

19. Piglansky et al. Bacteriologic and clinical efficacy of high dose amoxicillin for therapy of acute otitis media in children. Pediatr Infect Dis J. 2003 May;22(5):405-13.

20. Arguedas A et al. A randomized, multicenter, double blind, double dummy trial of single dose azithromycin versus high dose amoxicillin for treatment of uncomplicated acute otitis media. Pediatr Infect Dis J. 2005 Feb;24(2):153-61.

21. Marcy M, Takata G, Chan LS, Shekelle P, Mason W, Wachsman L, Ernst R, Hay JW, Corley PM, Morphew T, Ramicone E, Nicholson C. Management of acute otitis media. Evid Rep Technol Assess (Summ). 2000 Jun;(15):1-4.

22. Hytonen M, Atula T, Pitkaranta A. Complications of acute sinusitis in children. Acta Otolaryngol Suppl. 2000;543:154-7.

23. Voronkin VF, Sergeev MM, Perekhoda DL. Orbital and intracranial complications of paranasal sinus inflammation. Vestn Otorinolaringol. 1999;(4):25-7.

24. Gallagher RM, Gross CW, Phillips CD. Suppurative intracranial complications of sinusitis. Laryngoscope. 1998 Nov;108(11 Pt 1):1635-42.

25. Clayman GL et al. Intracranial complications of paranasal sinusitis: a combined institutional review. Laryngoscope. 1991 Mar;101(3):234-9. 
26. Singh B et al. Sinogenic intracranial complications. J Laryngol Otol. 1995 Oct;109(10):945-50.

27. Lerner DN et al. Intracranial complications of sinusitis in childhood. Ann Otol Rhinol Laryngol. 1995 Apr;104(4 Pt 1):288-93.

28. Chotmongkol V, Sangsaard S. Intracranial complications of chronic suppurative otitis media. Southeast Asian J Trop Med Public Health. 1992 Sep;23(3):510-3.

29. Long YT et al. Complications of otitis media requiring surgical intervention. Asian J Surg. 2002 Apr;25(2):170-4.

30. Kangsanarak J et al. Intracranial complications of suppurative otitis media: 13 years'experience. Am J Otol. 1995 Jan;16(1):104-9.

31. Berman S et al. Surgical management of uncomplicated otitis media in a pediatric Medicaid population. Ann Otol Rhinol Laryngol. 2000 Jul;109(7):623-7.

32. Pediatric Acute Otitis Media. San Diego: University of California.

33. Diagnosis and management of acute otitis media. American Academy of Family Physicians - Medical Specialty Society - American Academy of Pediatrics. 2004.

34. bronchomunal ${ }^{\circledR}$ - Farmabank 2005. Momento Medico S.r.1.

35. Schaad UB et al. Immunostimulation with OM-85 in children with recurrent infections of the upper respiratory tract: a double-blind, placebo-controlled multicenter study. Chest. 2002 Dec;122(6):2042-9.

36. Heikkinen T et al. Burden of influenza in children in the community. J Infect Dis. 2004 Oct 15;190(8):1369-73. Epub 2004 Sep 15.

37. Fagnani et al. Evaluation du retentissement économique et social des infections respiratoires et ORL récidivantes de l'enfant. Santé Publique 1995; 7 (3): 247-56.

38. Banz K et al. Economic evaluation of immunoprophylaxis in children with recurrent ear, nose and throat infections. Pharmacoeconomics. 1994 Nov;6(5):464-77.

39. Banche dati Istituto Nazionale di Statistica - www.istat.it.

40. Società Italiana di Nutrizione Umana - www.sinu.it.

41. Use of antibiotics in paediatric care. Singapore Ministry of Health - National Government Agency [Non-U.S.] 2002 Mar.

42. Viral upper respiratory infection(VURI) in adults and children. Bloomington(MN): Institute for Clinical Systems Improvement - Private Nonprofit Organization. 1994 Jun (revised 2004 May).

43. Kiivet RA et al. Drug use in Estonia in 1994-1995: a follow-up from 1989 and comparison with two Nordic countries. Eur J Clin Pharmacol. 1998 Apr;54(2):119-24.

44. Garattini L et al. Durata e costo delle visite in medicina generale: il progetto DYSCO Farmeconomia e percorsi terapeutici 2003; 4(2): 109-114.

45. Langley JM, Halperin S. Allergy to antibiotics in children: Perception versus reality. Paediatr Child Health. 2002; 7:233-237.

46. Diagnosis and treatment of otitis media in children. Institute for clinical system improvement, updated May 22, 2003.

47. Diagnosis and management of childhood otitis media in primary care. Scottish Intercollegiate Guidelines Network. Feb 2003.

48. Evidence based clinical practice guideline for medical management of acute otitis media in children 2 months to 13 years of age. Cincinnati Children's Hospital Medical Center - Hospital/Medical Center. 1999 (2004 Oct 29).

49. Diagnosis and treatment of acute otitis media. Dakota: the health care plan of the south-Dakota medical association.

50. No authors listed. Use of codeine- and dextromethorphan-containing cough remedies in children. Pediatrics. 1997 Jun;99(6):918-20.

51. Sore throat and tonsillitis. Finnish Medical Society Duodecim - Professional Association. 2001 Apr 22 (revised 2004 May 13). Various pagings.

52. Banche dati statistiche del Ministero della Salute - www.ministerosalute.it.

53. Delibera N'73-13176 del 26.04.2004 della Giunta Regionale del Piemonte - www.regione.piemonte.it

54. Drummond MF et al. Methods for the economic evaluation of health care programmes (2nd ed). Oxford: Oxford University Press, 1997. 